\title{
Musculoskeletal disorders and psychosocial risk factors among workers of the aircraft maintenance industry
}

\author{
Helen Cristina Nogueira ${ }^{\mathrm{a}^{*}}$; Ana Carolina Parise Diniz ${ }^{\mathrm{b}}$; Dechristian França Barbieri ${ }^{\mathrm{a}}$; Rosimeire \\ Simprini Padula ${ }^{\mathrm{c}}$; Rodrigo Luiz Carregaro ${ }^{\mathrm{d}}$ and Ana Beatriz de Oliveira, ${ }^{\mathrm{a}, \mathrm{b}}$ \\ ${ }^{a}$ Graduate Program in Physical Therapy, Universidade Federal de São Carlos UFSCar, São Paulo, Brazil \\ ${ }^{\mathrm{b}}$ Undergraduate Program in Physical Therapy, Universidade Federal de São Carlos UFSCar, São Paulo, Brazil \\ ' School of Physical Therapy, Universidade Federal de Mato Grosso do Sul (UFMS), Campo Grande, MS, Brazil. \\ ${ }^{\mathrm{d}}$ Masters in Physical Therapy Program, Universidade Cidade de São Paulo, São Paulo, Brazil
}

\begin{abstract}
During the recent decades Brazil has experienced an exponential growth in the aviation sector resulting in an increasing workforce. The aircraft maintenance industry stands out, where the workers have to handle different kind of objects. The aim of this study was to evaluate psychosocial indicators as well as musculoskeletal symptoms and disorders among aircraft maintenance workers. One hundred and one employees were evaluated $(32.69 \pm 8.25 \mathrm{yr}, 79.8 \pm 13.4 \mathrm{~kg}$, and $1.75 \pm 0.07 \mathrm{~m})$. Musculoskeletal symptoms and disorders were assessed through the Nordic Musculoskeletal Questionnaire (NMQ) and a standardized physical examination. The Job Content Questionnaire (JCQ) and the Utrecht Work Engagement Scale (UWES) were applied to evaluate psychosocial indicators. Results of the NMQ indicate the lower back as the most affected body region. On the other hand, the physical examination has shown clinical diagnosis of shoulder disorders. Neck, upper back and ankle/foot were also reported as painful sites. Most of workers have active work-demand profile and high work engagement levels. We suggest that musculoskeletal symptoms may be related to high biomechanical demand of the tasks performed by workers, what must be further investigated.
\end{abstract}

Keywords: Physical therapy, prevention; ergonomics;,WRMD, psychosocial indicators, aviation

\section{Introduction}

During the recent decades Brazil has experienced an exponential growth in the aviation industry. In 2005, civil aviation registered the higher productivity since the beginning of the decade. Data released by the National Union of Airway Companies show that, during 2009, more than thirty million passengers have traveled on domestic flights, which represents an increase of $19.4 \%$ in relation to data of 2004 . When looking at the last decade, the number of passengers flying on national companies increased by $146 \%$. It has moved from 22.8 million in 1998 to 56.2 million in 2008 [2].

Considering this growth, the aviation sector has considerably increased the number of workers employed by different industries from those dedicated to customer service until industries of cargo and aircraft maintenance. Subjects working in the aviation industry have a commitment with safety that gives to this sector a number of peculiarities related to attention, training and work engagement. Safety must be considered at all levels of the aviation industry. Nevertheless, safety stands out among air traffic controllers as well as among workers doing aircraft maintenance. Arvidsson et al [12] assessed the physical exposure, based on movements and electromyography recordings, among air traffic controllers when using two different information technology systems. The risk factors for work-related musculoskeletal disorders (WRMD) identified in their study were mainly related to the computer use and psychosocial factors present at the workplace. The literature does not have any information regarding musculoskeletal disorders and risk factors when the focus is the aircraft maintenance industry.

According to Ward et al [20], the aircraft maintenance industry is a complex system, where

*Corresponding author. E-mail: helencnogueira@yahoo.com.br 
organizational and work environment factors may contribute to produce a crash. Considering the large variability of activities developed by workers in this kind of industry, it is possible to highlight tasks involving manual material handling. Workers usually have to handle different objects ranging from small components to large pieces of the aircraft, while possible exposed to physical and psychosocial risk factors of the workplace. The association between manual material handling and the development of low back injury is well established in the literature $[4,24,17,30]$. Futhermore, manual material handling can also require great effort of upper limbs, lower limbs and neck $[17,30,22,25,5,1]$. The literature shows a large amount of information related to the low back loads during manual material handing while small effort has been applied on understanding loads on upper limbs [1]. Besides physical factors, psychosocial demands are reported as possible risk factors for musculoskeletal disorders on both the upper limbs and low back [28, 32]. Therefore, actions to control work-related musculoskeletal disorders may be based on a comprehensive analysis of the workplace, involving the assessment of both physical and psychosocial factors, as well as the musculoskeletal response resulting from the interaction between risk factors.

Considering the lack of knowledge available in the literature regarding risks factors of the aircraft maintenance industry as well as the importance of carrying out preventive actions, the aim of this study was to evaluate psychosocial indicators as well as musculoskeletal symptoms and WRMD among aircraft maintenance workers.

\section{Methods}

One hundred and one male employees (32.69 \pm 8.25 years, $79.8 \pm 13.4 \mathrm{~kg}, 175 \pm 7 \mathrm{~cm}$ ) of an aircraft maintenance company participated in this study. They do not perform maintenance itself (they are not aircraft mechanics) but their job gives support to the maintenance. Therefore all workers are used to perform manual material handling tasks - which were defined as tasks that require the use of force to carry, lift or lower objects with the hands. The study was approved by the local Ethics Committee on Human Research, and all participants signed an informed consent form.
Musculoskeletal complaints were recorded through the application of the Brazilian version of the Nordic Musculoskeletal Questionnaire (NMQ), which was validated by Barros and Alexandre [6]. A physical examination was also performed in order to investigate musculoskeletal disorders through the application of specific tests. It was based on the physical examination developed by the Department of Occupational and Environment Medicine of Lund University [15], which focus on the assessment of the upper limbs. Considering that workers are also exposed to risks involving the lower back, specific tests for the lower back were also included in the examination protocol.

Psychosocial indicators were evaluated through the Job Content Questionnaire (JCQ) and the Utrecht Work Engagement Scale (UWES). The JCQ, proposed by Theorell in 1988 [21], is based on the demand-control model, and it also assesses social support. The UWES focuses on the work engagement, investigating positive aspects of the work instead of assessing burnout, as the majority of psychosocial scales and questionnaires.

The workers were initially introduced to the study and received information about the questionnaires. They could subsequently fill in the questionnaire in the presence of the researchers. The physical examination was schedule and performed individually on all workers.

The data were processed according to the instruction of each questionnaire. The physical examination has allowed the establishment of diagnosis of upper limb disorders, based on standardized criteria [15]. The results of tests for low back region were reported separately. All data were descriptively presented.

\section{Results}

Data of musculoskeletal symptoms recorded using the Nordic Musculoskeletal Questionnaire are presented in Table 1. It was observed that $77.2 \%$ $(n=78)$ of the workers have had complaints during the last 12 months. For the last seven days, 55.4\% $(n=56)$ of the participants had reported pain in some part of their body.

The NMQ data show that the complaints are mainly concentrated on lower back, neck, upper back in both annual and weekly prevalence. The shoulder region also has presented high percentage of reported symptoms. Nevertheless, it occurs when 
the last year is considered rather than the previous week. The elbows have shown the smallest rate of reported symptoms.

Table 1

Weekly and annual prevalence of musculoskeletal symptoms, percentage of workers who were prevented from carrying out normal duties in the last 12 months and percentage of workers who sought a health professional in the last 12 months. Data obtained through the NMQ

\begin{tabular}{lcccc} 
& \multicolumn{2}{c}{ among employees of aircraft maintenance. } \\
BODY SITES & Annual Prevalence & Weekly Prevalence & $\begin{array}{c}\text { Functional incapacity } \\
\text { Search for medical } \\
\text { assistance }\end{array}$ \\
\cline { 2 - 6 } & $\mathbf{( \% )}$ & $\mathbf{( \% )}$ & $\mathbf{( \% )}$ \\
\hline Neck & 38.46 & 20.19 & 10.58 & \\
Shoulders & 36.54 & 10.58 & 4.81 & 15.38 \\
Upper Back & 37.50 & 14.42 & 9.62 & 1.92 \\
Elbows & 3.85 & 0.00 & 18.27 & 10.58 \\
Lower Back & 45.19 & 26.92 & 3.85 & 1.92 \\
Wrist/hands & 23.08 & 8.65 & 4.81 & 4.08 \\
Hip/Thighs & 16.35 & 5.77 & 8.65 & 5.73 \\
Knees & 25.00 & 11.54 & 6.73 & 9.62 \\
Ankles/Foot & 30.77 & 17.31 & & 8.65 \\
\hline
\end{tabular}

The percentage of employees that have experienced functional incapacity during the last 12 months was less than $37 \%(n=37)$. The body part most frequently associated with functional incapacity was the lower back $(18.3 \%)$, followed by neck (10.6\%) and upper back (9.6\%). Approximately $45.5 \%(n=46)$ of employees have searched for medical assistance as a result of musculoskeletal symptoms. The most common region associated with search for medical assistance was also the lower back (23.1\%). In general, it is possible to identify a pattern where the lower back is the most frequently affected region, followed by neck and upper back. When symptoms during the last 12 months are considered, the shoulders also stand out. This distinction is not so strong for the weekly prevalence as well as for functional incapacity or search for medical assistance. In these conditions the ankles/foot were also highly reported.

The data regarding the diagnosis established on the basis of the standardized physical examination and criteria are presented in Table 2.

Table 2

Percentage of cases (diagnosis established with no right and left distinction), percentage of bilateral cases (diagnoses established for both right and left body regions) and percentage of workers with diagnosis.

\begin{tabular}{|c|c|c|c|c|}
\hline Diagnosis & & Cases $(\%)$ & Bilateral Cases (\%) & Workers with diagnosis (\%) \\
\hline \multirow{3}{*}{$\begin{array}{l}\text { 己̌ } \\
\ddot{Z}\end{array}$} & Tension neck syndrome & 14.74 & - & 14.74 \\
\hline & Cervical syndrome & 7.37 & - & 7.37 \\
\hline & Thoracic outlet syndrome & 8.42 & - & 8.42 \\
\hline \multirow{4}{*}{$\begin{array}{l}\frac{\dot{0}}{0} \\
\frac{\overrightarrow{0}}{\tilde{D}}\end{array}$} & Supraspinatus tendinitis & 5.26 & 1.05 & 4.21 \\
\hline & Infraspinatus tendinitis & 8.42 & 2.11 & 6.32 \\
\hline & Bicipital tendinitis & 12.63 & 4.21 & 8.42 \\
\hline & Acromioclavicular syndrome & 17.89 & 4.21 & 13.68 \\
\hline \multirow{5}{*}{ 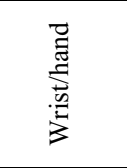 } & De Quervain's disease & 8.42 & 1.05 & 7.37 \\
\hline & Carpal tunnel syndrome & 4.21 & 0.00 & 4.21 \\
\hline & Pronator teres syndrome & 1.05 & 0.00 & 1.05 \\
\hline & Ulnar nerve entrapment at the elbow & 2.11 & 1.05 & 1.05 \\
\hline & Ulnar nerve entrapment at the wrist & 1.05 & 0.00 & 1.05 \\
\hline
\end{tabular}

In general, data indicate a relatively small number of workers with diagnosis of musculoskeletal disorder. The most common disorders are associated with tension in the neck and shoulder muscles.
The data presented in Table 3 shows the percentage of workers reporting symptoms on the lower back, according to the physical examination. 
Table 3

Percentage of workers with complaints and percentage of bilateral cases related to the lower back.

\begin{tabular}{lcc} 
Symptom & \% workers & $\begin{array}{c}\text { \% bilateral } \\
\text { cases }\end{array}$ \\
\hline Pain & 48.42 & - \\
Pain radiating to the legs & 10.53 & 4.21 \\
Numbness & 12.63 & 9.47 \\
Weakness & 6.32 & 5.26 \\
\hline
\end{tabular}

According to the tests performed during the physical examination of the lower back (trunk movements - tilting, rotation, flexion-extension; muscle strength test; esthesiometry) none positive signals were found among the subjects. However, approximately $50 \%$ of workers have reported lower back pain. Other symptoms (as numbness or weakness in the legs) were reported by less than $13 \%$ of the participants (Table 3 ).

The results of the JCQ are presented in Figure 1. It is observed that most of the workers $(34 \%)$ was classified as active according to the demand-control model (high psychological demand and high control over the work process). The assessment of social supported showed that $57.4 \%$ of the participants have high level of social interaction with superiors and colleagues.

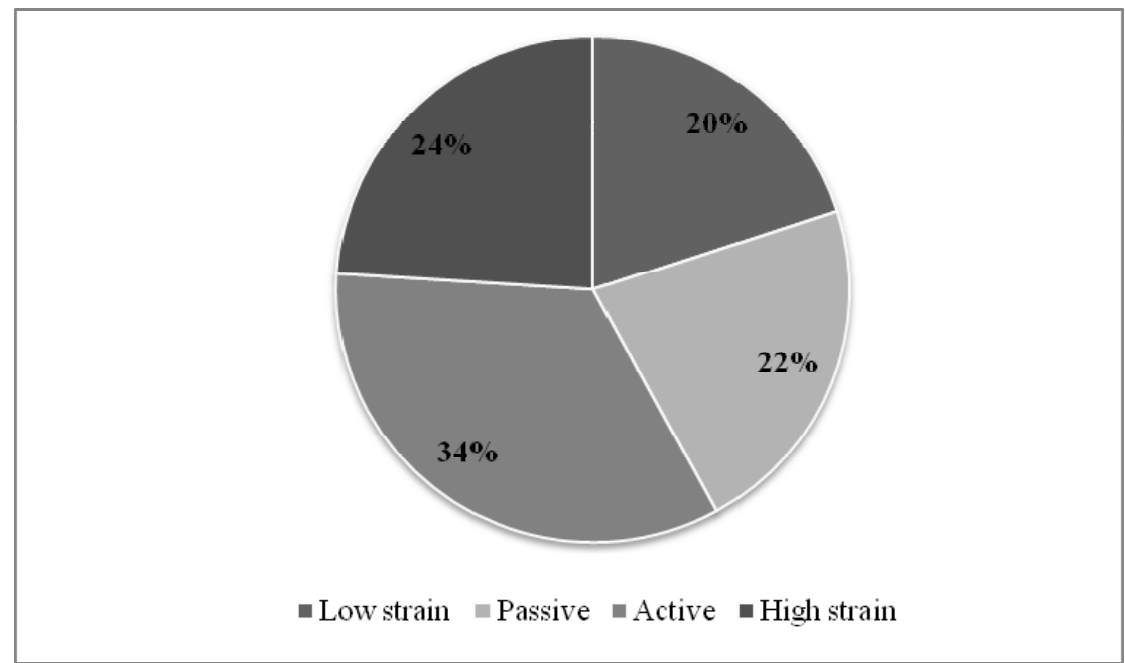

Figure 1. Percentage of workers classified in each profile of the demand-control model, defined according to the Job Content Questionnaire.

Table 4 shows data of the UWES. The 'high' level was the most common classification for all the domains of the scale (engagement, absorption, dedication and vigor). No worker was classified as "very low" level for the domains engagement and vigor, and less than $2 \%$ of the participants had shown "very low" level of dedication and absorption. 
Table 4

UWES data among workers: percentages of workers classified in each level ('very low', 'low', 'medium', 'high' and 'very high') for vigor, dedication, absorption and engagement domains.

\begin{tabular}{|c|c|c|c|}
\hline Domain | level & $\%$ of workers & Domain | level & \% of workers \\
\hline Vigor & & Absorption & \\
\hline Very low & 0.00 & Very low & 0.96 \\
\hline Low & 5.77 & Low & 2.88 \\
\hline Medium & 20.19 & Medium & 35.58 \\
\hline High & 42.31 & High & 37.50 \\
\hline Very high & 31.73 & Very high & 23.08 \\
\hline Dedication & & Work Engagement & \\
\hline Very low & 1.92 & Very low & 0.00 \\
\hline Low & 10.58 & Low & 4.81 \\
\hline Medium & 29.81 & Medium & 27.88 \\
\hline High & 47.12 & High & 44.23 \\
\hline Very high & 10.58 & Very high & 23.08 \\
\hline
\end{tabular}

\section{Discussion}

Musculoskeletal symptoms in neck, shoulder and lower back are strongly associated with high levels of workload with subsequent absenteeism. The expenses generated by musculoskeletal imbalances have great socioeconomic impact due to the decrease in productivity and early retirements $[8$, 11]. Investigations focusing on both physical and psychosocial aspects are important to identify the installation of WRMD and to design effective preventive measures, according to the major cause of musculoskeletal disorders [3]. The aviation industry has a growing workforce, and the lack of knowledge available in the literature regarding risk factors for WRMD in this sector impairs the development of actions that aim to control the development of musculoskeletal disorders.

Kemp et al [26] evaluated the civilian and military U.S. Air Force (USAF), and found that handling aircraft components, boxes and furniture were the activities that most frequently caused injury to workers $(33 \%)$, resulting a high number of absences. It shows the importance of looking at manual material handling tasks. On the other hand, Hoozemans et al [23] evaluated 10 workers handling boxes and they did not find any symptom on the lower back among them. When evaluating manual material handling tasks it is important to consider the height of the task and the weight of the load these features directly affect the compression force on structures of the lower back. In the aircraft maintenance scenario the high mass of the objects handled can overload the lumbar spine. Besides that, other body segments than the lower back are strained during manual material handling [1]. These aspects corroborate the results shown by the NMQ in this study. We observed that the lower back was the most common region where pain occurred during the past 12 months (45\%) and during the last week $(25 \%)$. Symptoms on this region were associated with functinal impairment as well as with search for medical assistance. Besides the lower back, symptoms on neck, upper back, shoulders and ankles/foot were also reported. Strain on shoulders during manual material handling has been reported in the literature $[1,14]$. It occurs mainly when the handling task is performed either in high or low surfaces [1]. The trapezius insertion between neck and shoulder and the effort of this muscle during shoulder movements can explain neck symptoms it is difficult to differentiate neck and shoulder segments due the connection established by the trapezius muscle [15]. In general, the standing position is used all over the day, and it explains symptoms reported on ankles and foot. The safety shoe used also contributes to the development of symptoms on foot since they are not comfortable.

The most common disorders diagnosed through the physical were the acromioclavicular syndrome $(17.9 \%)$, neck tension syndrome $(14.7 \%)$, and bicipital tendinitis (12.6\%), which do not fully agree with the results of the NMQ. The differences between the findings obtained by the questionnaire and the physical examination are related to the specificity of the last one. The standardized musculoskeletal examination was developed to evaluate neck and upper extremity disorders [15]. Therefore, this examination does not include the assessment of the lower back. Even though the repetitive and static muscular work has increased among jobs (causing strain on neck and upper limbs), the lower back is highly impaired among 
workers doing heavy work. It shows the need for standardized diagnostic methods to assess the lower back. Further studies must focus on this topic. The assessment of the lower back is not an easy task considering the difficulty to identify the source of pain in clinical examination. In the upper limbs, the superficial structures allow palpation and it facilitates the establishment of the clinical diagnosis. The deep structures that are source of pain in the lower back are not accessible to palpation, which makes the examination difficult.

In general, musculoskeletal symptoms recorded using the NMQ were higher than disorders diagnosed by the physical examination. It may indicate the occurrence of strain on specific body sites that can lead to WRMD to medium and long term. In order to identify factors of the workplace that are related to this strain, the evaluation of the workplace is essential.

The literature shows that the combination of physical and psychosocial workplace risk factors can increase musculoskeletal complaints in relation to the exposure to one or the other kind of risk factors [13]. Pransky et al [9] justify the importance of considering psychosocial factors when determining how workers perceive and report musculoskeletal symptoms. In addition, besides being risk factors for developing musculoskeletal disorders, poor psychosocial conditions are associated with poor quality of activities performed by workers $[28,33]$. The presence of high demand and high control at work may have an inverse relationship with musculoskeletal disorders, especially with lower back, neck and shoulder involvements $[18,19,31]$. The results recorded by the JCQ and UWES, in general, indicate good psychosocial conditions, with the majority of workers classified as active (according to the demand-control model) and with high scores of engagement, vigor, dedication, and absorption at work. Nevertheless, Joling et al [3] suggest that psychosocial conditions are not good enough to ensure lower incidence of musculoskeletal symptoms, which corroborates our results. Good psychosocial indicators have not prevented the development of musculoskeletal symptoms among the participants. The social context of the work is also an important factor that affects psychosocial scores, having a direct relationship with musculoskeletal symptoms $[27,11,10]$. Once most of workers $(57.4 \%)$ have shown high scores of social support, this aspect may not be associated with musculoskeletal symptoms and disorders identified among the workers.

Furthermore, not all psychosocial indicators are inversely related with WRMD. In a meta-analysis study, Crawford et al [7] divided the psychological demand at work into two aspects. The first one is the demand that offers autonomy for the decision making and creates personal growth and satisfaction in the worker, despite the possible requirements such as time pressure and high levels of responsibility at work. The second aspect is the demand faced when dealing with obstacles of organizational and political conflicts. It impairs or delays the development of tasks, stresses the workers and has a negative correlation with work engagement. It is possible that this negative aspect of the demand is present in the aircraft maintenance industry investigated. However, high scores of work engagement do not support this hypothesis. Marras [32] suggests that psychosocial stress is more likely to influence spine loading at low levels of work intensity than at high levels of biomechanical demand. In this case, workers can show good psychosocial indicators and still report complaints, particularly considering the lower back. It indicates that physical factors of the workplace can be associated with the results of WRMD found among the participants.

Finally, the prediction of musculoskeletal disorders has high correlation with the presence of previous symptoms. The probability of recurrence of injury is related with the great vulnerability of the affected tissues, increased sensitivity to pain and permanent exposure to risk factors at the workplaces $[29,16]$. When considering psychosocial aspects, the low-quality in communication shows the highest correlation with the development of WRMD [3]. Moreover, the higher the psychological demand at work, the higher the risk of stress and absenteeism. Job resources increase engagement and reduce the risk of stress $[18,7]$

Workers of the aircraft maintenance industry involved with manual material handling tasks are exposed to good psychosocial conditions, which does not prevent WRMD since the report of pain and functional incapacity was high and particularly associated with the lower back. It indicates the presence of physical risk factors of the workplaces that must be investigated. 


\section{References}

[1] A.B. Oliveira, L.C.C.B Silva, H.J.C.G. Coury. How do low/high height and weight variation affect upper limb movements during manual material handling of industrial boxes? Brazilian Journal of Physical Therapy, 2011; ahead of print: DOI: 10.1590/S1413-35552011005000017 15(4).

[2] Brasil, 2010. Ministério da Defesa. Acessado em 16/03/2010. availabe in: http://www.anac.gov.br/estatistica/estatisticas1.asp.

[3] C. I Joling, B.M, Blatter, J. F. Ybema, P. M. Bongers. Can favorable psychosocial work conditions and high work dedication protect against the occurrence of work-related musculoskeletal disorders? Scand J Work Environ Health, 2008; 34(5):345-355.

[4] D.B. Chaffin, K.S. Park. A longitudinal study of low-back pain as associated with occupational weight lifting factors. American Industrial Hygiene Association Journal, 1973; 34(12): 513-25.

[5] D.R. Smith, N. Wei, L. Zhao, R.S. Wang. Musculoskeletal complaints and psychosocial risk factors among Chinese hospital nurses. Occupational Medicine, 2004; 54: 579-582.

[6] E.N.C. Barros, N.M.C. Alexandre. Cross-cultural adaptation of the Nordic Musculoskeletal Questionnaire. International Nursing Review, 2003; 50: 101-108.

[7] E. R. Crawford, J. A. LePine, B. L. Rich. Linking Job Demands and Resources to Employee Engagement and Burnout: A Theoretical Extension and Meta-Analytic Test. Journal of Applied Psychology, 2010; 95:5, 834-848.

[8] G. Armon, S. Melamed, A. Shirom, I. Shapira. Elevated Burnout Predicts the Onset of Musculoskeletal Pain Among Apparently Healthy Employees. Journal of Occupational Health Psychology, 2010; 15( 4): 399-408.

[9] G. Pransky, T.B. Snyder, J. Himmelstein. The organizational response Influence on Cumulative Trauma Disorders in the Workplace. Beyond Byomechanics: Psychosocial Aspects of Musculoskeletal Disorders in Office Work, 2005; (15):219228.

[10]G.A.M. Ariens, W. van Mechelen, P.M. Bongers, L.M. Bouter, G. van der Wal. Psychosocial risk factors for neck pain: a systematic review. Am J Ind Med, 2001; 39:180-93.

[11]G. J. Macfarlane, N. Pallewatte, P. Paudyal, F. M. Blyth, D. Coggon, G. Crombez, S. Linton, P. Leino-Arjas, A. J. Silman, R. J. Smeets, D. van der Windt. Evaluation of workrelated psychosocial factors and regional musculoskeletal pain: results from a EULAR Task ForceAnn Rheum Dis, 2009; 68:885-891.

[12] Arvidsson, G.A. Hansson, S.E. Mathiassen, S. Skerfving. Changes in physical workload with implementation of mouse-based information technology in air traffic control. International Journal of Industrial Ergonomics, 2006; 36:613622.

[13]J.J. Devereux, I.G. Vlachonikolis, P.W. Buckle. Epidemiological study to investigate potential interaction between physical and psychosocial factors at work that may increase the risk of symptoms of musculoskeletal disorder of the neck and upper limb, Occup Environ Med 2002;59:269277.

[14]K. Søgaard, B. Laursen, B.R. Jensen, G. Sjøgaard. Dynamic loads on the upper extremities during two different floor cleaning methods. Clinical Biomechanics, 2004;16(10): 86679.

[15]K. Ohlsson, R.G. Attewell, B. Johnsson, A. Ahlm, S. Skerfving, An assessment of neck and upper extremity disorders by questionnaire and clinical examination. Ergonomics, 1994; 37:891-897.
[16]L. Hestbaek, Leboeuf-Yde C, Kyvik KO. Is comorbidity in adolescence a predictor for adult low back pain?: a prospective study of a young population. BMC Muskuloskelet Disord, 2006;16:7-29.

[17]L.M. Straker. An overview of manual handling injury statistics in Western Australia. International Journal of Industrial Ergonomics, 1999; 24 (4): 357-64.

[18]J.D. Nahrgang, D.A. Hofmann, F.P. Morgeson, Safety at Work: A Meta-Analytic Investigation of the Link Between Job Demands, Job Resources, Burnout, Engagement, and Safety Outcomes. Journal of Applied Psychology, 2011; 96(1):71-94.

[19]M. Coetzee, M. Villiers, Sources of job stress, work engagement and career orientations of employees in a South African fi nancial institution. Southern African Business Review Volume, 2010;14(1): 27-58.

[20] M. Ward, N. Mc Donald, R. Morrison, D. Gaynor, T. Nugent. A performance improvement case study in aircraft maintenance and its implications for hazard identification. Ergonomics, 2010; 53(2): 247-267.

[21] M.G.M. Alves, D. Chor, E. Faerstein, C.S. Lopes, G.L. Werneck. Versão resumida da "job stress scale": adaptação para o português. Revista de Saúde Pública, 2004; 38(2): 164-171.

[22] M.J. Lusted, C.L. Carasco, J.A. Mandryk, S. Heatey. Self reported symptoms in the neck ad upper limbs in nurses. Applied Ergonomics, 1996; 27(6): 381-387.

[23] M.J.M. Hoozemans, I. Kingma, W.H.K. Vries, J.H. Dieen. Effect of lifting height and load mass on low back loading. Ergonomics, . 2008; 51(7): 1053-1063.

[24] M.M. Ayoub. Problems and solutions in manual materials handling: the state of art. Ergonomics, 1992; 35(7-8): 713-28.

[25]N.N. Menzel, S.M. Brooks, T.E. Bernard, A. Nelson. The physical workload of nursing personnel: association with musculoskeletal discomfort. International Journal of Nursing Studies, 2004; 41: 859-867.

[26]P.A. Kemp, B.R. Burnham, B. Copley, M.J, Shim. Injuries to Air Force Personnel Associated with Lifting, Handling, and Carrying Objects. Am J Prev Med, 2010; 38(1S):S148-S155.

[27]R.A. Nicholas, M. Feuerstein, S. Suchday. Workstyle and upper extremity symptoms: a biobehavioral perspective. J Occup Environ Med. 2005;47(4):352-61.

[28]S. Thorn. Muscular activity in light manual work - with reference to the development of muscle pain among computer users. Doctoral Thesis.Chalmers University of Technology, Göteborg, Sweden. 2005.

[29] S. Ijmker. Risk factors for arm-wrist-hand and neck-shoulder symptoms among office workers. Amsterdam: Body@Work, Free University Amsterdam; 2008.

[30] S.S. Yeung, A. Genaidy, J. Deddens, A. Alhemood, P.C. Leung. Prevalence of musculoskeletal symptoms in single and multiple body regions and effects of perceived risk of injury among manual handling workers. Spine, 2002; 27(19): 2166-71.

[31]T. Theorell. Possible mechanisms behind the relationship between the demand-control support model and disorders of the locomotor system. Beyond Byomechanics: Psychosocial Aspects of Musculoskeletal Disorders in Office Work, 2005; (4):56-63. 2005.

[32] W.S. Marras. Occupational low back disorder causation and control. Ergonomics, 2000a; 43(7): 880-902.

[33] W.S. Marras, K.G. Davis, C.A. Heaney, A.B. Maronitis, W.G. Allread. The Influence of Psychosocial Stress, Gender, and Personality on Mechanical Loading of the Lumbar Spine. Spine, 2000b; 23: 3045-3054. 